\title{
Model success is not the end of the matter
}

Geoff Brumfiel, Washington

The standard model of high-energy physics received a ringing endorsement last week, as researchers revealed the most accurate measurements so far taken of an effect that may explain the surplus of matter in the Universe.

The results, presented at the International Conference on High Energy Physics in Amsterdam by teams from the United States and Japan, focus on a fundamental effect that some think could explain why we exist.

Physicists believe that at the time of the Big Bang, the Universe contained equal amounts of matter and antimatter. But these should have annihilated each other, leaving no matter to create galaxies, stars - or us. One explanation for the glut of matter in the Universe is an effect called chargeparity $(\mathrm{CP})$ violation, which causes matter and antimatter to behave in slightly different ways.

Two groups - the Belle collaboration based at the High Energy Accelerator Research Organization in Tsukuba, Japan, and BaBar at the Stanford Linear Accelerator Center in the United States have measured the decay of particles called $\mathrm{B}$ mesons and their antiparticle equivalent, anti-B mesons. Both teams detected a slight difference in the decay rates of the particles, which is interpreted as evidence for $\mathrm{CP}$ violation.

The measurements, which are around twice as accurate as those previously taken (see Nature 412, 105; 2001), agree well with each other and also with the standard model's predictions. "The agreement is as perfect as it could be," says Stewart Smith, a physicist at Princeton University and spokesman for the BaBar collaboration.

Despite this support for the standard model, current measures of $\mathrm{CP}$ violation show that the effect is not strong enough to account for the excess of matter. "The measurement fails by a factor of ten billion or so," Smith says. "There's something out there that we don't know about yet. These latest measurements don't even give a hint."

Some physicists believe that a possible explanation could be the existence of superheavy particles that are not predicted by the standard model. The presence of these particles in the early Universe would have influenced the balance between matter and antimatter, tipping the scales in the favour of matter.

Delegates in Amsterdam were due to hear tentative evidence for super-heavy particles this week. The g-2 experiment at the Brookhaven National Laboratory in New York state measures a property of particles called muons that should deviate slightly
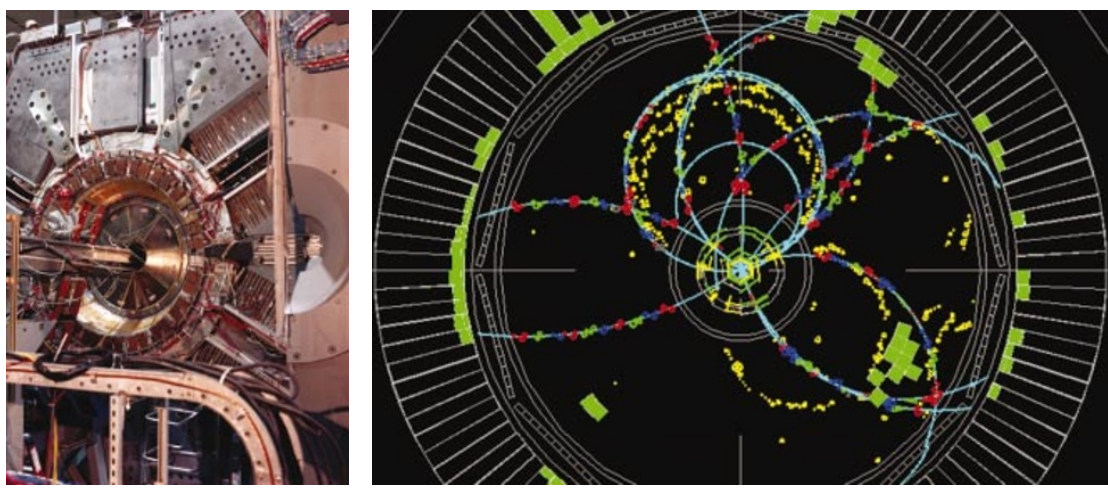

Data on particle decays (right) obtained using BaBar's equipment vindicate physics' standard model.

from the predictions of the standard model if super-heavy particles do indeed exist. The latest results from Brookhaven hint at this deviation, but physicists will treat the results

with care after a similar announcement las year turned out to be based on a flawed calculation (see Nature 410, 291; 2001 and Nature 415, 6; 2002).

\section{'Superbug' hurdles key drug barrier \\ Helen Pearson \\ DNA sequence analysis revealed that the}

Microbiologists have expressed fears that hospitals are ill-equipped to cope with 'superbugs', after a bacterium resistant to one of the last lines of antibiotic defence was identified in the United States.

The US Centers for Disease Control and Prevention (CDC) in Atlanta, Georgia, reported last month that a strain of vancomycin-resistant Staphylococcus aureus had been isolated in June from the foot ulcer and catheter of a patient in Michigan.

S. aureus often causes severe infections in patients' wounds, and outbreaks can lead to whole hospital wards being shut down.

Vancomycin became a vital weapon against $S$. aureus when strains resistant to the preceding antibiotic of choice, methicillin, emerged in the 1980s. But since 1987, when relatively harmless gut bacteria called enterococci evolved resistance to vancomycin, microbiologists have been warning that these genes could be transferred to $S$. aureus.

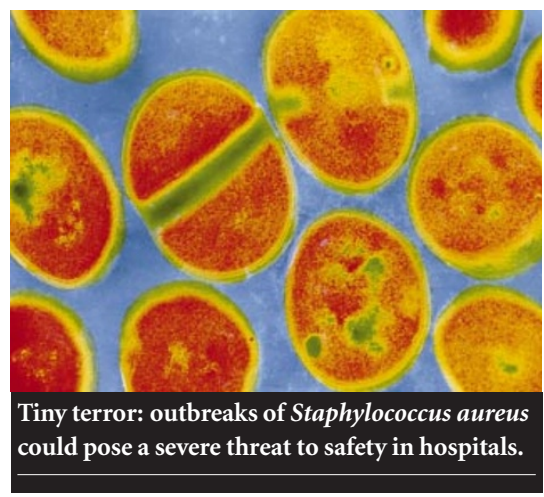
Michigan S. aureus strain did acquire its resistance genes from an enterococcus. A vancomycin-resistant Enterococcus faecalis isolated from the same patient could explain the jump, suggests the CDC's Fred Tenover, who led the analysis.

The outbreak is probably the first of many, as vancomycin-resistant enterococci are increasing in prevalence - almost a third of enterococci in US intensive-care units are now resistant to the antibiotic. Careful monitoring and rigorous control of outbreaks are the best response, says Gary French, a microbiologist at Guy's \& St Thomas' Hospital in London.

Drug-resistant bacteria are usually detected in the lab by their ability to grow near paper discs soaked in antibiotic. But this method can fail with vancomycin, as it does not diffuse well, giving unusual patterns of bacterial growth that lab staff may not recognize. A back-up test, in which bacteria are grown on plates soaked in vancomycin, may not be used because of the costs involved.

If there is a repeat of the Michigan incident, French fears that some hospitals lack the staff and isolation rooms to control the infection. "We know what we ought to do but I'm concerned that there aren't enough resources to do it," he says.

Vancomycin resistance is not as worrying as it would have been before the introduction of new antibiotics called linezolid and quinupristin/dalfopristin in 1999-2000, when vancomycin was often the last line of defence. But clinical biologists are keen to limit the use of these newer drugs, as some $S$. aureus strains are already resistant to them. 